\title{
Diagnosis of HPV-driven head and neck cancer with a single test in routine clinical practice
}

Haïtham Mirghani ${ }^{1}$, Odile Casiraghi ${ }^{2}$, Furrat Amen ${ }^{3}$, Mingxiao $\mathrm{He}^{4}$, Xiao-Jun $\mathrm{Ma}^{4}$, Patrick Saulnier ${ }^{5}$, Ludovic Lacroix ${ }^{5}$, Françoise Drusch ${ }^{6}$, Aïcha Ben Lakdhar ${ }^{2}$, Jean Lacau Saint Guily ${ }^{7}$, Cécile Badoual ${ }^{8}$, Jean Yves Scoazec ${ }^{2,5,6}$ and Philippe Vielh ${ }^{2,5,6}$

${ }^{1}$ Department of Otolaryngology-Head and Neck Surgery, Gustave Roussy Cancer Campus, Villejuif, France; ${ }^{2}$ Department of Biopathology, Gustave Roussy Cancer Campus, Villejuif, France; ${ }^{3}$ Department of Otolaryngology, Peterborough City Hospital and Addenbrooke's Hospital, Cambridge, UK; ${ }^{4}$ Advanced Cell Diagnostics, Hayward, CA, USA; ${ }^{5}$ Laboratory of Translational Research, Gustave Roussy Cancer Campus, Villejuif, France; ${ }^{6}$ Biobank, Gustave Roussy Cancer Campus, Villejuif, France; ${ }^{7}$ Department of Otolaryngology-Head and Neck Surgery, Hôpital Tenon, Assistance Publique-Hôpitaux de Paris, Paris, France and ${ }^{8}$ Department of Pathology, Hôpital Européen Georges Pompidou, Assistance Publique-Hôpitaux de Paris, Paris, France

\begin{abstract}
Accurate screening of HPV-driven head and neck squamous cell carcinoma is a critical issue. Although there are commercial direct and indirect assays for HPV-related head and neck squamous cell carcinoma, none are ideal. Recently, a novel RNA in situ hybridization test (the RNAscope HPV-test) has been developed for the detection of high-risk HPV E6/E7 mRNA in formalin-fixed paraffin-embedded tissue. However, validation of this assay against the 'gold standard' (identification of high-risk HPV E6/E7 mRNA in fresh-frozen tissue by quantitative real-time (qRT)-PCR) has only been reported by one team. Formalin-fixed paraffin-embedded samples from 50 patients with tonsil or tongue base carcinoma were tested using the RNAscope HPV-test, p16 immunohistochemistry, and chromogenic in situ hybridization for high-risk HPV-DNA. The results were compared with those of qRT-PCR on matched fresh-frozen samples. Compared with the reference test, the sensitivity, specificity, positive, and negative predictive values of the RNAscope HPV-test and of p16 immunohistochemistry were $93 \%, 94 \%, 96 \%$, $88 \%$ and $96 \%, 93 \%, 96 \%$, and $93 \%$, respectively. Five cases were discrepant between the RNAscope HPV-test and p16-immunohistochemisrty. The RNAscope HPV-test demonstrated excellent analytical performance against the 'gold standard' and is easier to interpret than chromogenic in situ hybridization. p16-immunohistochemistry also performed very well, however its main weakness is that it is an indirect marker of the presence of HPV. These data suggest that the RNAscope HPV-test is a promising test that could be developed as a clinical standard for the precise identification of HPV-driven oropharyngeal squamous cell carcinoma.
\end{abstract}

Modern Pathology (2015) 28, 1518-1527; doi:10.1038/modpathol.2015.113; published online 25 September 2015

Accurate screening of HPV-driven head and neck squamous cell carcinoma is a critical issue. HPV status is currently used as a prognostic marker and to interpret the outcomes of clinical trials involving oropharyngeal squamous cell carcinoma, but in the near future HPV status may have a more important role in deciding treatment and follow-up of head and neck squamous cell carcinoma. Currently, the gold standard assay to detect these cancers is based on the

Correspondence: Dr H Mirghani, Department of OtolaryngologyHead and Neck Surgery, Gustave Roussy Cancer Campus, 114 Rue Edouard Vaillant, 94805 Villejuif, France.

E-mail: haitham.mirghani@gustaveroussy.fr

Received 18 June 2015; accepted 11 August 2015; published online 25 September 2015 identification of viral oncogenes mRNA (E6/E7) by reverse-transcriptase-PCR (RT-PCR) in fresh frozen samples. ${ }^{1,2}$ However, this technique is not useful for routine screening as it is technically demanding.

Several other methods have been developed, ${ }^{3}$ to directly or indirectly identify HPV-related head and neck squamous cell carcinoma, but none of them are ideal (Table 1). Detection of HPV-DNA can be performed by PCR or in situ hybridization. PCR is highly sensitive but lacks specificity, as it cannot distinguish between an infection truly involved in carcinogenesis and a bystander oral HPV infection,,$^{3,4}$ which is not uncommon in the general population. ${ }^{5}$ On the other hand, detection of HPV-DNA by in situ hybridization has good specificity, as the virus is directly visualized within the tumor cell nuclei, 
Table 1 Advantages and drawbacks of currently available HPV detection methods

\begin{tabular}{lll}
\hline Detection technique & Advantages & Drawbacks \\
\hline p16 immunostaining & High sensitivity & Low specificity \\
& Feasible on FFPE material & Indirect (surrogate) marker of viral activity \\
& Provides proof of transcriptional activity & through cell cycle dysregulation \\
& Available commercial kit with CE certification & No exclusive link, between HPV infection, p16 \\
& Simple and accessible to most laboratories & over-expression, and carcinogenesis
\end{tabular}

HPV-DNA in situ hybridization

Consensual HPV PCR (end point PCR (qualitative))

HPV-type-specific qRT-PCR

E6/E7 mRNA PCR (RT-PCR)
High specificity

Feasible on FFPE material

Provides visual detection of infected cells (preservation of tissue context)

Distinction between episomal and integrated

Available commercial kit with CE certification

Simple and accessible to most laboratories

High sensitivity

Feasible on FFPE material

Targets numerous oncogenic HPV strains

Available commercial kit with CE certification

Cost effective and adequate for routine screening

in most laboratories

High sensitivity

Feasible on FFPE material

Provides quantitative measure of viral load
Reduced sensitivity when low viral loads No information about transcriptional activity Technically difficult to interpret

Requires DNA extraction

No direct evidence of transcriptional activity Risk of contamination and detection of HPV from surrounding healthy tissue

Requires DNA extraction

No direct evidence of transcriptional activity

Risk of contamination and detection of HPV from surrounding healthy tissue

No validated threshold for viral load interpretation

Requires fresh or frozen material Risk of RNA degradation

Inadequate for routine screening
Detects clinically relevant infections with evidence of active oncogene transcription Considered as the 'gold standard'

Abbreviation: CE, Conformité Européene; HPV, human papillomavirus; qRT-PCR, quantitative real-time-PCR.

but several authors have noted a lack of sensitivity. ${ }^{6,7}$ Assessment of p16 protein expression by immunohistochemistry is widely used to define HPV status. Indeed, numerous studies have shown a strong correlation between p16 overexpression and HPVdriven oropharyngeal squamous cell carcinoma. ${ }^{8,9}$ Moreover, it is a cost-effective and practical tool that can be used routinely in most histopathological laboratories. ${ }^{3}$ Despite these advantages, p16immunohistochemestry is not reliable enough to be used as a standalone test as its performance may be confounded by a lack of specificity for oncogenic HPV infection. ${ }^{3}$ Several authors have reported that $\sim 8-20 \%$ of p16-positive oropharyngeal squamous cell carcinomas do not contain HPV-DNA and have a molecular profile that is closer to HPV-negative cancers. ${ }^{10-12}$

In this context, determining the presence and activity of HPV with a single test is very attractive. The RNAscope HPV-test (Advanced Cell Diagnostics, Hayward, CA, USA) is a recent in situ hybridization technique that detects E6/E7 mRNA from up to 18 high-risk HPVs (HPV16, 18, 26, 31, 33, 35, 39, 45, 51, $52,53,56,58,59,66,68,73$, and 82 ). This new assay is in commercial development but is not yet available for clinical use. It uses formalin-fixed paraffin-embedded (FFPE) tissue sections and the interpretation is visual with light microscopy, the transcripts being directly identified within the tumor. These features make the RNAscope HPV-test a potentially ideal tool for routine laboratory testing. However, before its adoption as a routine screening test can be advocated, it must be assessed independently by several teams. To date, only Schache et $a 1^{13}$ have compared its performance with the gold standard test and have concluded that the RNAscope HPV-test demonstrates excellent analytical and prognostic performance.

Using a cohort of well-characterized oropharyngeal squamous cell carcinomas, comprising matched fresh-frozen and formalin-fixed paraffin-embedded tumor samples, we sought to validate the RNAscope HPV-test against quantitative RT (qRT)-PCR for HPV16, 18, and 33 E6/E7 transcripts.

\section{Materials and methods}

\section{Patients and Tumor Samples}

Tumor samples from 50 patients with tonsil or base of tongue squamous cell carcinoma were retrieved from the Gustave Roussy Cancer Center tissue bank. These samples were randomly selected from those for which matched fresh frozen and formalin-fixed 
paraffin-embedded tissues were available. All samples were collected before treatment. Medical files of each patient were retrospectively analyzed by the investigators including clinical and pathological reports. Our Institutional Review Board approved the study protocol and all patients provided written informed consent.

\section{Preparation of Sections from Formalin-Fixed Paraffin-Embedded Tumor Tissue Blocks}

Paraffin sections were prepared according to the sandwich method. That is to say the first and last sections were stained by hematoxylin and eosin to check for tumor presence. The remaining $4 \mu \mathrm{m}$ sections were used to perform p16 immunohistochemistry, HPV-DNA in situ hybridization and the RNAscope HPV test.

\section{p16 Immunohistochemistry}

Representative 4- $\mu \mathrm{m}$ whole tumor formalin-fixed paraffin-embedded tissues sections were deparaffinized and subjected to antigen retrieval using $10 \mathrm{~mm}$ citrate buffer $\left(92^{\circ} \mathrm{C}\right.$ for $\left.30 \mathrm{~min}\right)$. p16 immunohistochemistry was carried out with a proprietary kit (CINtec Histology; MTM laboratories AG) on a Ventana Benchmark autostainer (Ventana Medical Systems, Tucson, AZ, USA). A tonsil squamous cell carcinoma with high p16 expression was used as a positive control. The primary antibody was omitted from negative controls. p16 immunohistochemistry was scored as positive if there was strong and diffuse nuclear and cytoplasmic staining present in $>70 \%$ of the malignant cells. ${ }^{14}$ All other staining patterns were scored as negative.

\section{Detection of High-Risk HPV DNA by In Situ Hybridization}

DNA in situ hybridization was performed on representative 4- $\mu \mathrm{m}$ whole tumor formalin-fixed paraffin-embedded sections from each case using the ISH I View Blue Plus Detection Kit (Ventana Medical System) according to the manufacturer's instructions. The assay used the Ventana HPV III Family 16, Probe B, a cocktail recognizing the highrisk (HR) HPV types 16, 18, 31, 33, 35, 45, 51, 52, 56, 58, 59, 68, and 70. Ventana Red Counterstain II (Ventana Medical System) was used. Controls in each run included a known HPV16-positive oropharyngeal squamous cell carcinoma case (positive control) and a section of normal tonsil (negative control). Positive staining was identified as blue nuclear dots. Any definitive nuclear staining in the tumor cells was considered positive. Cases were classified in a binary manner as either positive or negative.

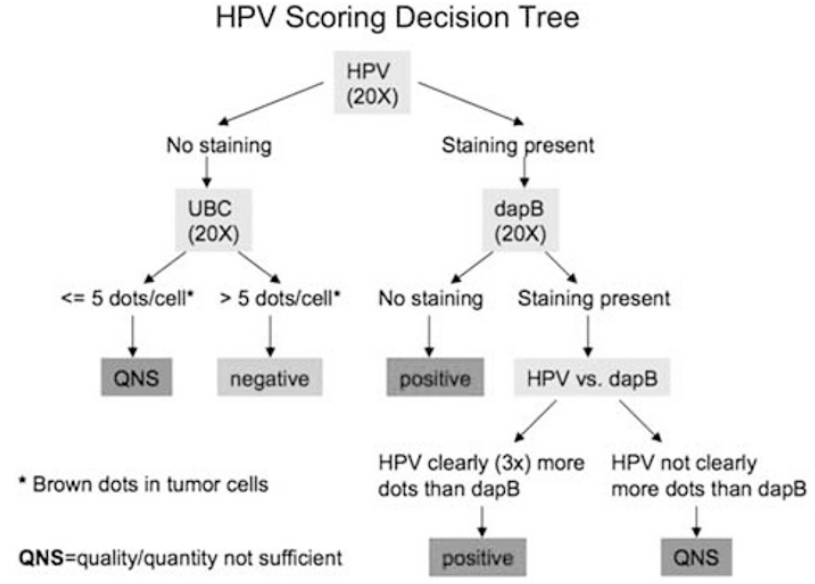

Figure 1 HPV scoring decision tree: every case was stained with three probes: (1) Ubiquitin $C$ (UBC), an endogenous housekeeping gene used as positive control and an indicator of sample RNA integrity, (2) $\operatorname{dapB}$ (diaminopimelate B), a bacterial gene used as negative control, and (3) HPV-HR18, a cocktail probe for 18 highrisk types. Slides are scored according to the following scoring algorithm to determine if a sample is HPV positive or HPV negative: (A) If high-risk-HPV stain is higher than dapB signal (if any), the sample is HPV positive. (B) If HR-HPV signal is absent or similar to that of dapB, the Ubiquitin $C$ slide is examined. If the Ubiquitin $\mathrm{C}$ probe detects $>5$ dots/cell indicating sufficient RNA quality in the sample, the sample is scored as negative for HRHPV. Otherwise (Ubiquitin $\mathrm{C}<5$ dots/cell), the sample is disqualified due to insufficient RNA quality.

\section{Detection of High-Risk HPV E6/E7 mRNA by RNA In Situ Hybridization (RNAscope HPV-Test)}

Detection of high-risk-HPV E6/E7 mRNA was performed using the RNAscope 2.0 BROWN assay kit and the HPV-HR18 probe cocktail (Advanced Cell Diagnostics) in accordance with the manufacturer's instructions. In brief, $4 \mu \mathrm{m}$ sections were deparaffinized and pretreated with heat and protease before hybridization with target-specific probes for the E6 and E7 genes of 18 HR-HPV genotypes (HPV18, 26, $31,33,35,39,45,51,52,53,56,58,59,66,68,73$, and 82). Ubiquitin $C$ (a constitutively expressed endogenous gene) and the bacterial gene, $\operatorname{dap} B$ (diaminopimelate $\mathrm{B}$ ), were used as positive and negative controls, respectively.

The Ubiquitin $\mathrm{C}$ test was used to assess the presence of hybridizable RNA to confirm adequate RNA quality and was defined as adequate if there were at least five punctate signal dots in the majority of cells in the section. This is especially important when the HPV probe signal is negative to avoid a false-negative result. The dapB test was used to assess non-specific staining, only those cases that were negative or weakly stained were considered for HPV scoring. A positive HPV test result was defined as punctate staining that localized to the cytoplasm and/or nucleus of any of the malignant cells, and where staining was present in the negative control, it was at least three times as strong as the dapB staining (Figure 1). 


\section{Review of the Miscroscopic Slides}

The RNAscope HPV-test slides were scored independently by two authors (Mingxiao He and Odile Casiraghi) and the p16 immunohistochemistry and high-risk HPV DNA-in situ hybridization tests were also assessed independently by two authors (Odile Casiraghi and Aïcha Ben Lakdhar). Scoring systems were based on a binary classification (positive vs negative). All the people involved in theses microscopic slides review were blinded from the results of all the different assays that were tested (including the qRT-PCR, described in the next paragraph) and from the outcomes from the other reviewers.

The results were collated, and discordant scores were resolved at a meeting between the pathologists to establish a consensus interpretation.

\section{Total RNA Extraction from Fresh Frozen Sections}

Quantification of tumor cell proportion within all fresh frozen samples was undertaken to ensure that extracted RNA was representative of tumor rather than surrounding stroma or inflammatory cells. Total RNA was isolated from fresh frozen tissue by using Trireagent (Sigma-Aldrich), and was purified on RNeasy columns (Qiagen) according to the manufacturer's protocol. RNA quality, based on the 28S:18S ribosomal RNA ratio, was assessed with an Agilent bioanalyzer (Agilent Technologies, Palo Alto, CA, USA). All specimens included in this study had a ratio $>1.5$. RNA. Samples were frozen in nuclease-free water until use (Promega Corporation).

\section{RT and qRT-PCR Analysis to Identify HPV 16/18/33 E6 mRNA}

Reverse transcription was performed using $1 \mathrm{mg}$ total RNA from each sample in a reaction volume of $20 \mu \mathrm{l}$ containing $50 \mathrm{U}$ Moloney murine leukemia virus reverse transcriptase, $20 \mathrm{U}$ ribonuclease inhibitor (Applied Biosystems, Foster City, CA, USA), $1 \mathrm{mmol} / \mathrm{l}$ dNTPs (Amersham Pharmacia Biotech, NJ, USA), $5 \mathrm{mmol} / \mathrm{l} \mathrm{MgCl}_{2}, 10 \mathrm{mmol} / \mathrm{l}$ Tris-HCl (pH 8.3), $10 \mathrm{mmol} / \mathrm{l} \mathrm{KCl}$, and $50 \mathrm{pmol} / \mathrm{l}$ random hexamers (Perkin Helmer Corp PE, Applied Biosystems, Thermo Fischer Scientific).

Duplicate real-time RNA (cDNA) PCR reactions were carried out with the same primer and probe combinations for the HPV16 E6 gene under identical conditions, as described by Schache et $a l,{ }^{7}$ on the Applied Biosystems 7500 FAST System. Primers and a FAM-MGB-labeled TaqMan probe were designed to specifically amplify the HPV18 E6 region (synthesized by Applied Biosystems) and optimized with the cervical cell line HeLa (UK Health Protection Agency Culture Collections-93021013). A commercially available HPV33 detection system (Primer Design HPV33 Kit) ${ }^{7}$ was used to analyze HPV33 E6 gene expression. Both HPV18 and HPV33 expression analyses were conducted on the Applied Biosystems 7500 FAST System. Standard curves were done to test the efficiency of the reaction with each set of primer-probes. Plasmid solution specific to HPV16/ 18/33 were serially diluted, to obtain a quantification gradient of 500, 5000, $50000,500000,5000000$ viral copies, and run in parallel with each samples. Each sample was tested in duplicate. Unspecific amplification was checked by a melting curve at the end of each PCR. Human VIC-MGB-labeled ACTB primers and probe (Applied Biosystems) were incorporated as an endogenous control to facilitate internal normalization and relative gene expression quantification for all expression assays. This PCR reaction was run in parallel with each sample, using a standard curve based on a commercial RNA (Universal Human Reference RNA, catalog \#740000, Stratagene) converted in cDNA in parallel with samples.

The mean of duplicate reactions was used to calculate relative gene expression by the 2-DDCT method. ${ }^{15}$ The qRT-PCR assays were performed by Patrick Saulnier who was blinded to the outcomes of the microscopic slides.

\section{Results}

\section{Population Description}

Thirty-six men and 14 women were included in our study. Location, TNM staging, age, tobacco consumption, and histological features are summarized in Table 2. The tumor cell proportion, within freshfrozen and formalin-fixe paraffin-embedded samples, is reported in Table 3 .

\section{Test Analysis}

Outcomes for the four detections assays are summarized in Table 3 .

Data for p16 immunohistochemistry and the RNAscope HPV-test, were available for the whole cohort, whereas data for HPV-DNA in situ hybridization was available for 48 out of 50 patients. qRT-PCR for HR-HPV E6 mRNA detection (the reference test in our study) was available in 43 out of 50 patients due to insufficient RNA quality or quantity in seven fresh-frozen samples.

The scoring of the RNAscope HPV-test and p16 slides were completely concordant between the pathologists. Discordant cases were only seen with the HR-HPV DNA in situ hybridization assay and were resolved at a meeting to establish a consensus interpretation.

High-risk HPV E6 mRNA detection by qRT-PCR was positive in 27 (63\%) samples and negative in $16(37 \%)$ samples. Amongst the positive samples, HPV16 was found in 22 cases (82\%), HPV18 in 2 cases (7\%), and HPV33 in 3 (11\%) cases.

The RNAscope HPV-test was positive in 33 samples (66\%) and negative in 17 (34\%) samples 
Table 2 Population description

\begin{tabular}{|c|c|c|c|c|}
\hline & Age/sex & Location & $T N M$ & Tobacco \\
\hline 1 & $60 / \mathrm{F}$ & Tonsil & T2N2C & Yes \\
\hline 2 & $67 / \mathrm{M}$ & Tonsil & $\mathrm{T} 4 \mathrm{aN} 2 \mathrm{c}$ & Yes \\
\hline 3 & $62 / \mathrm{M}$ & Tonsil & T3N1 & Yes \\
\hline 4 & $70 / \mathrm{M}$ & Tonsil & T3N0 & Yes \\
\hline 5 & $67 / \mathrm{M}$ & Tongue base & T3N0 & No \\
\hline 6 & $60 / \mathrm{M}$ & Tongue base & $\mathrm{T} 4 \mathrm{aN} 2$ & Yes \\
\hline 7 & $55 / F$ & Tonsil & T3N1 & Yes \\
\hline 8 & $67 / \mathrm{M}$ & Tonsil & T3N0 & Yes \\
\hline 9 & $57 / \mathrm{M}$ & Tongue base & T3N2c & Former \\
\hline 10 & $59 / \mathrm{M}$ & Tonsil & $\mathrm{T} 2 \mathrm{~N} 2 \mathrm{~b}$ & Yes \\
\hline 11 & $55 / \mathrm{M}$ & Tonsil & T4aNo & Yes \\
\hline 12 & $65 / \mathrm{M}$ & Tongue base & T4aN2c & Yes \\
\hline 13 & $65 / \mathrm{M}$ & Tonsil & T2N2c & Yes \\
\hline 14 & $57 / \mathrm{M}$ & Tonsil & T2N3 & Yes \\
\hline 15 & $61 / \mathrm{M}$ & Tonsil & T2N3 & No \\
\hline 16 & $41 / \mathrm{F}$ & Tonsil & T2No & Yes \\
\hline 17 & $63 / \mathrm{M}$ & Tongue base & T2N2c & No \\
\hline 18 & $78 / \mathrm{M}$ & Tonsil & $\mathrm{T} 3 \mathrm{Nx}$ & Yes \\
\hline 19 & $59 / \mathrm{F}$ & Tonsil & $\mathrm{T} 2 \mathrm{~N} 2 \mathrm{~b}$ & No \\
\hline 20 & $59 / \mathrm{F}$ & Tonsil & T1N2b & No \\
\hline 21 & $72 / \mathrm{F}$ & Tonsil & T3N2b & No \\
\hline 22 & $61 / \mathrm{M}$ & Tonsil & T3N2b & Yes \\
\hline 23 & $60 / \mathrm{M}$ & Tongue base & T4aNo & Yes \\
\hline 24 & $60 / \mathrm{M}$ & Tongue base & T2N0 & No \\
\hline 25 & $71 / \mathrm{M}$ & Tongue base & $\mathrm{T} 2 \mathrm{~N} 2 \mathrm{~b}$ & former \\
\hline 26 & $61 / \mathrm{M}$ & Tonsil & $\mathrm{T} 3 \mathrm{~N} 2 \mathrm{~b}$ & no \\
\hline 27 & $61 / \mathrm{F}$ & Tongue base & T2N1 & no \\
\hline 28 & $77 / \mathrm{M}$ & Tonsil & T3N2c & no \\
\hline 29 & $60 / \mathrm{M}$ & Tongue base & T1N2b & Yes \\
\hline 30 & $58 / \mathrm{F}$ & Tongue base & T1N2c & Former \\
\hline 31 & $64 / \mathrm{M}$ & Tongue base & T3N2b & Yes \\
\hline 32 & $52 / \mathrm{M}$ & Tongue base & $\mathrm{T} 2 \mathrm{~N} 2 \mathrm{~b}$ & Yes \\
\hline 33 & $73 / \mathrm{F}$ & Tonsil & T4aN2b & no \\
\hline 34 & $65 / F$ & Tongue base & T4aN3 & Yes \\
\hline 35 & $65 / F$ & Tongue base & T2N2c & no \\
\hline 36 & $42 / \mathrm{F}$ & Tongue base & T4N2b & no \\
\hline 37 & $46 / \mathrm{M}$ & Tonsil & T1N2b & Former \\
\hline 38 & $63 / \mathrm{F}$ & Tongue base & $\mathrm{T} 4 \mathrm{~N} 1$ & Former \\
\hline 39 & $54 / \mathrm{M}$ & Tonsil & T2No & Yes \\
\hline 40 & $55 / \mathrm{M}$ & Tongue base & T4aN2a & Yes \\
\hline 41 & $45 / \mathrm{M}$ & Tongue base & $\mathrm{T} 4 \mathrm{aN} 2 \mathrm{c}$ & Yes \\
\hline 42 & $69 / \mathrm{M}$ & Tongue base & $\mathrm{T} 2 \mathrm{~N} 2 \mathrm{~b}$ & Former \\
\hline 43 & $69 / \mathrm{M}$ & Tonsil & $\mathrm{T} 2 \mathrm{~N} 2 \mathrm{~b}$ & Yes \\
\hline 44 & $55 / \mathrm{M}$ & Tonsil & T3N2b & No \\
\hline 45 & $50 / \mathrm{M}$ & Tonsil & $\mathrm{T} 4 \mathrm{aN} 2 \mathrm{~b}$ & No \\
\hline 46 & $57 / \mathrm{M}$ & Tongue base & $\mathrm{T} 2 \mathrm{~N} 2 \mathrm{~b}$ & No \\
\hline 47 & $50 / \mathrm{M}$ & Tongue base & $\mathrm{T} 2 \mathrm{~N} 2 \mathrm{~b}$ & Yes \\
\hline 48 & $70 / \mathrm{F}$ & Tonsil & T3N0 & Yes \\
\hline 49 & $64 / \mathrm{M}$ & Tongue base & T2N2a & Yes \\
\hline 50 & $46 / \mathrm{M}$ & Tongue base & T2N2a & Yes \\
\hline
\end{tabular}

(Figure 2). Outcome comparisons between this assay and the reference test was concordant in 40 out of 43 samples $(93 \%)$. Fifteen HPV-positive cases with the RNAscope HPV-test showed some minor staining $(\sim 1-2$ dots in $<10 \%$ cells $)$ on the negative control probe slides, but the HPV probe slides showed overwhelmingly stronger signals (i.e., much more numerous and bigger signal dots in a majority of malignant cells), which was unambiguously scored. The fact that the two independent pathologists were $100 \%$ concordant indicated that this minor staining background does not interfere with interpretation. p16 immunohistochemistry was positive in 34 $(68 \%)$ samples and negative in $16(32 \%)$ samples. There were two $(4.6 \%)$ discrepant cases between p16 immunohistochemistry and the reference test, and $5(10 \%)$ cases between p16 immunohistochemistry and the RNAscope HPV-test.

Eight $(16 \%)$ cases were discrepant between the RNAscope HPV-test and HPV-DNA in situ hybridization. In five cases the RNAscope HPV-test was positive and the HPV-DNA in situ hybridization was negative. In 3 out of 5 cases both the reference test and p16-immunohistochemistry were positive and supported the RNAscope HPV-test outcomes. p16 immunohistochemistry or the reference test were positive for each of the two other cases. The RNAscope HPV-test was negative and HPV-DNA in situ hybridization positive in three cases and all of them were negative with both the reference test and p16 immunohistochemistry.

The sensitivity, specificity, positive, and negative predictive values of the RNAscope HPV-test against the identification of high-risk HPV E6/E7 mRNA by qRT-PCR were 93\%, 94\%, 96\%, and $88 \%$, respectively. The sensitivity, specificity, positive, and negative predictive values for p16 against the reference test were $96 \%, 93 \%, 96 \%$, and $93 \%$, respectively. Performance of HPV DNA in situ hybridization against the reference test was poorer with a sensitivity, specificity, positive, and negative predictive value of $76 \%, 75 \%, 83 \%$, and $70 \%$, respectively (Table 4).

\section{False-Positive and False-Negative Reporting}

The RNAscope HPV-test yielded positive results for one case $(2.3 \%)$ where there was an absence of detectable HPV mRNA by qRT-PCR. Corresponding test results for this case indicated that p16 immunohistochemistry was negative and HR-HPV DNA in situ hybridization was positive.

The RNAscope HPV-test classified as negative two cases $(4.6 \%)$ that displayed evidence of HPV-transcripts with qRT-PCR (HPV16 in one case, HPV33 in the other). Both of these cases were also classified as negative by high-risk-HPV DNA in situ hybridization but they were positive with p16 immunohistochemistry.

Five cases out of 50 (10\%) were discordant between the RNAscope HPV-test and p16 immunohistochemistry. Two samples $(4 \%)$ were positive with the RNAscope HPV-test and negative with p16 immunohistochemistry, and the three others (6\%) were negative with the RNAscope HPV-test and positive with p16 immunohistochemistry. All five samples were also tested with qRT-PCR. Among the two cases that were positive with the RNAscope HPV-test and negative with p16 immunohistochemistry, the qRT-PCR found that one sample was positive and the other negative. For the three cases that were negative with the RNAscope HPV-test 
Table 3 Tests results

\begin{tabular}{|c|c|c|c|c|c|}
\hline & $\begin{array}{l}\text { \% tumor cells in FFPE } \\
\text { (fresh frozen) samples }\end{array}$ & $q R T-P C R$ & RNAscope HPV-test & p16 IHC & $D N A-I S H$ \\
\hline 1 & $40(70)$ & Negative & Negative & Negative & Negative \\
\hline 2 & $90(70)$ & Negative & Negative & Negative & Negative \\
\hline 3 & $50(80)$ & Negative & Negative & Negative & Negative \\
\hline 4 & $70(80)$ & Negative & positive & positive & Negative \\
\hline 5 & $60(80)$ & Positive (++, HPV16) & Positive & Positive & Positive \\
\hline 6 & $80(90)$ & Positive (+++, HPV33) & Positive & Positive & Negative \\
\hline 7 & $90(70)$ & Negative & Negative & Negative & Negative \\
\hline 8 & $30(30)$ & Negative & Negative & Negative & Negative \\
\hline 9 & $80(60)$ & Positive (+, HPV16) & Positive & Positive & Positive \\
\hline 10 & $90(90)$ & Positive (+++, HPV18) & Positive & Positive & Positive \\
\hline 11 & $70(60)$ & Negative & Negative & Negative & Positive \\
\hline 12 & $30(60)$ & Negative & Negative & Negative & Negative \\
\hline 13 & $80(50)$ & Negative & Negative & Negative & Positive \\
\hline 14 & $80(50)$ & Negative & Negative & Positive & Negative \\
\hline 15 & $90(90)$ & Positive (+++, HPV16) & Positive & Positive & Positive \\
\hline 16 & $80(40)$ & Positive (++, HPV16) & Positive & Positive & Positive \\
\hline 17 & $70(80)$ & Positive (+, HPV16) & Positive & Positive & Positive \\
\hline 18 & $90(90)$ & Negative & Negative & Negative & Negative \\
\hline 19 & 70 & NA & Positive & Positive & Positive \\
\hline 20 & 80 & NA & Positive & Positive & Positive \\
\hline 21 & 80 & NA & Positive & Positive & Positive \\
\hline 22 & $80(90)$ & Negative & Negative & Negative & Negative \\
\hline 23 & 60 & Negative & Negative & Negative & NA \\
\hline 24 & 70 & NA & Positive & Positive & Positive \\
\hline 25 & $80(80)$ & Positive (+++, HPV33) & Positive & Positive & Positive \\
\hline 26 & 70 & Positive (+++, HPV16) & Positive & Positive & Positive \\
\hline 27 & 60 & Positive (+, HPV 16) & Positive & Positive & Positive \\
\hline 28 & 70 & Positive (+++, HPV18) & Positive & Positive & NA \\
\hline 29 & $70(90)$ & Negative & Positive & Negative & Positive \\
\hline 30 & $80(90)$ & Negative & Negative & Negative & Negative \\
\hline 31 & $90(80)$ & Positive $(+++$, HPV16) & Positive & Positive & Positive \\
\hline 32 & $60(70)$ & Positive (+, HPV16) & Positive & Positive & Positive \\
\hline 33 & $80(80)$ & Positive (++, HPV16) & Positive & Positive & Positive \\
\hline 34 & $70(80)$ & Positive (+, HPV 33) & Negative & Positive & Negative \\
\hline 35 & 70 & NA & Positive & Positive & Positive \\
\hline 36 & 70 & NA & Positive & Positive & Positive \\
\hline 37 & $40(30)$ & Positive (+, HPV16) & Positive & Positive & Positive \\
\hline 38 & $90(90)$ & Positive (+++, HPV16) & Positive & Positive & Positive \\
\hline 39 & $80(90)$ & Positive (+++, HPV16) & Positive & Positive & Positive \\
\hline 40 & $60(80)$ & Positive $(+++$, HPV16) & Positive & Positive & Positive \\
\hline 41 & $80(70)$ & Negative & Negative & Negative & Positive \\
\hline 42 & $70(90)$ & Positive (+++, HPV16) & Positive & Positive & Positive \\
\hline 43 & $80(50)$ & Positive $(+++$, HPV16) & Positive & Positive & Positive \\
\hline 44 & $70(80)$ & Positive (+++, HPV16) & Positive & Positive & Positive \\
\hline 45 & $50(70)$ & Positive (+, HPV16) & Positive & Positive & Positive \\
\hline 46 & $40(60)$ & Positive (+, HPV16) & Positive & Positive & Negative \\
\hline 47 & $80(70)$ & Positive (++, HPV16) & Positive & Positive & Negative \\
\hline 48 & $40(60)$ & Negative & Negative & Negative & Negative \\
\hline 49 & $60(90)$ & Positive (+, HPV16) & Positive & Negative & Negative \\
\hline 50 & $80(80)$ & Positive (++, HPV16) & Negative & Positive & Negative \\
\hline
\end{tabular}

Abbreviations: IHC, immunohistochemistry; ISH, in situ hybridization; NA, not available; qRT-PCR, quantitative real-time-PCR.

Relative gene expression quantification: +(low), ++(intermediate), and +++(high).

and positive with p16 immunohistochemistry, the qRT-PCR was negative in two cases and positive in one case.

\section{Discussion}

HPV-driven oropharyngeal squamous cell carcinomas are on the rise in Western countries. ${ }^{16,17}$ These tumors represent a distinct subgroup within head and neck squamous cell carcinoma, which are traditionally related to tobacco and alcohol abuse, and have a clearly favorable prognosis. ${ }^{18,19}$ These differences should lead to tailored medical care in the coming years. However, this objective will not be fully realized without better testing modalities to accurately identify them. Indeed, there is no widely recognized consensus on HPV testing for head and neck squamous cell carcinoma and the currently available tests have important weaknesses. ${ }^{3}$

As both the initiation and maintenance of an HPVdriven carcinoma requires persistent viral oncogene 

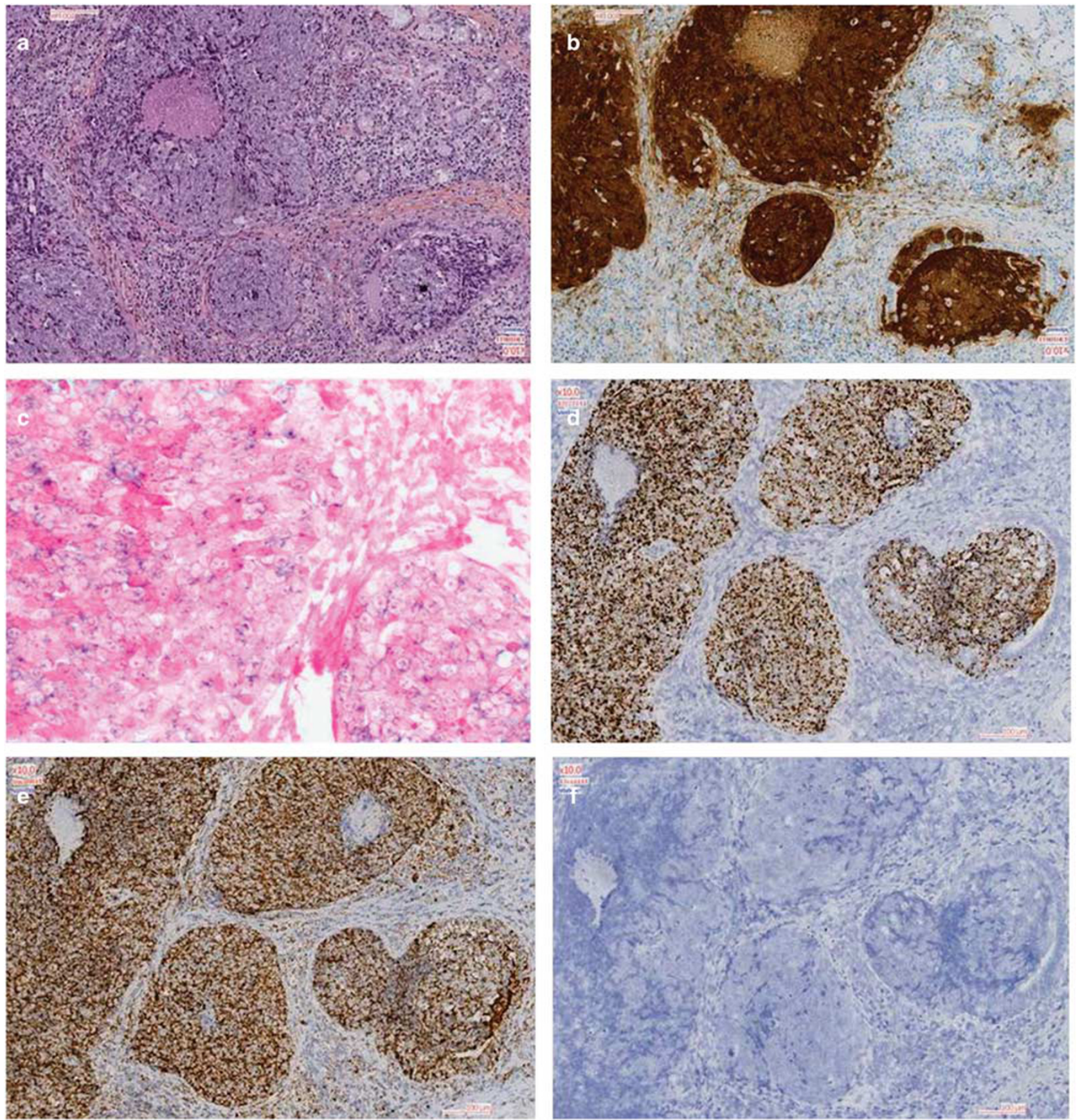

Figure 2 Photomicrographs of an oropharyngeal squamous cell carcinoma with positive RNA in situ hybridization and p16 immunohistochemistry. (a) Hematoxylin and eosin morphology, (b) positive p16 immunohistochemistry, (c) equivocal HPV-DNA in situ hybridization due to non-specific background staining, (d) positive RNA in situ hybridization with punctate brown staining, (e) Ubiquitin C (positive control to confirm that RNA quality is sufficient), and (f) dapB (negative control).

Table 4 Diagnostic capabilities of individual tests by comparison with the reference test

\begin{tabular}{lcccc}
\hline Diagnostic test & Sensitivity (\%) & Specificity (\%) & Positive predictive value (\%) & Negative predictive value (\%) \\
\hline RNAscope & 93 & 94 & 96 & 88 \\
P16 IHC & 96 & 93 & 96 & 93 \\
HPV DNA ISH & 76 & 75 & 83 & 70 \\
\hline
\end{tabular}


expression, the detection of E6/E7 viral oncogenes transcripts (E6/E7 mRNA) by RT-PCR has been proposed as the most appropriate 'gold standard' or reference test. ${ }^{1,2,20}$ However this is not feasible as part of the routine diagnostic process because it is technically cumbersome and requires, ideally, fresh-frozen tissue which is a fragile and a scarce resource. ${ }^{3,20}$ In this context, testing for HR-HPV E6 and E7 transcripts by RNA in situ hybridization in formalin-fixed paraffin-embedded tissue is particularly appealing.

In our study, we found that the RNAscope HPVtest has a high analytical accuracy when compared with the gold standard. Indeed, the sensitivity, specificity, positive, and negative predictive value were respectively of $93,94,96$ and $88 \%$. Schache et $a l,{ }^{13}$ who are to date the only ones to have performed a similar comparison in a cohort of 79 patients, also reported very high-scores highlighting that the RNAscope HPV-test's good outcomes are reproducible. In their study, the RNAscope HPV-test had a sensitivity of $97 \%$ and a specificity of $93 \%$ against the reference test, with positive and negative predictive values of $91 \%$ and $98 \%$, respectively. Recently, Gao et $a 2^{21}$ have developed a novel qRTPCR method for quantification of HPV transcripts in archived tissues. They have noted that qRT-PCR and RNAscope HPV-test were 100\% concordant in determining HPV status in a series of 45 OPSCC. Although in this study qRT-PCR was not performed in fresh-frozen tissue but in formalin-fixed paraffinembedded samples, these results are concordant with our findings and the findings of Schache et al. ${ }^{13}$

Among our three discrepant cases, one was classified as positive with the RNAscope HPV-test but displayed no evidence of HPV transcripts with qRT-PCR. This was the case for three out of four discrepant cases in Schache's series. ${ }^{13}$ These discordant results can be explained by the fact that the RNAscope HPV-test targets 18 high-risk HPV's whereas the RT-PCR assays, used in both the current and Schache et $a l^{13}$ studies, are restricted to the three most common genotypes found in oropharyngeal squamous cell carcinomas (HPV16, 18, and 33). If this assumption is correct, this implies that the RNAscope HPV-test's true performances are even better than what we have observed.

Conversely, the RNAscope HPV-test classified as negative two of our cases that displayed evidence of HR-HPV transcripts with qRT-PCR. Schache et al ${ }^{13}$ reported one similar case. The reasons behind this are still unknown but there are several possible scenarios. This could be due to a lack of sensitivity of the RNAscope HPV-test in samples with a low expression of E6/E7 mRNA, which was the case for one of our two samples and that of Schache et al, ${ }^{13}$ but other explanations are possible including the fact that pre-analytical factors such as fixation and processing may have resulted in suboptimal preservation of the target molecules in these three samples.

A few authors have compared the RNAscope HPVtest to p16 expression by immunohistochemistry and to HPV-DNA detection by in situ hybridization. ${ }^{14,22,23}$ Although none of these assays represent the reference test, these studies are interesting because p16 immunohistochemistry and HPV-DNA in situ hybridization are the most frequently used methods to identify HPVdriven oropharyngeal squamous cell carcinomas. ${ }^{3}$

Our comparison of the RNAscope HPV-test with HPV-DNA in situ hybridization favored the RNAscope HPV-test as expected. Indeed, several studies have confirmed that the sensitivity of HPV-DNA in situ hybridization is suboptimal, ranging from 79 to $82 \%$, and that non-specific background signals can hamper outcome interpretation in a significant number of cases. ${ }^{3,6,7}$ This was probably the case for the samples number 11 and 41 that we considered as positive. Indeed, these samples tested negative with the three other assays suggesting that they were actually HPV-DNA in situ hybridization false positive. As the transcription of viral mRNA provides a natural amplification step (cells contain more viral transcripts than viral genomes) this may contribute to the increased sensitivity of the RNAscope HPVtest because of a higher number of targets. Another key advantage of the RNAscope assay over conventional chromogenic in situ hybridization methods is that it specifically amplifies the hybridization signals without amplifying the background noise. ${ }^{24}$

In common with previous studies, we found a high correlation between p16 immunohistochemistry and the RNAscope HPV-test as only five cases were discordant. ${ }^{14,22,23}$ It is currently well established that p16 overexpression is a very strong surrogate marker to identify HPV-driven oropharyngeal squamous cell carcinomas as it indicates deregulation of the Retinoblastoma pathway by HR-HPV E7 oncoprotein. Owing to its numerous advantages, p16 immunohistochemistry (Table 1) has emerged as a key test to define HPV status and prognosis in a clinical setting. ${ }^{3,8}$ However, it is clearly acknowledged that $\sim 8-20 \%$ of p16-positive oropharyngeal squamous cell carcinomas are HPV-negatives ${ }^{10-12}$ and this is even more problematic in other upper aerodigestive anatomical subsites, where p16's positive predictive value drops significantly. ${ }^{25}$ This highlights the absence of a direct and exclusive link between HPV infection, p16 over-expression, and carcinogenesis and suggests that non-viral mechanisms may have a role in p16 over-expression. This lack of specificity is the main weakness of this assay, although we found p16 immunohistochemistry performed very well in our cohort. This might be explained by the fact that we enrolled only tonsillar squamous cell carcinoma (which is the oropharyngeal subsite that is the most significantly associated with HPV-induced malignancy) and that we employed very stringent criteria to define p16-positivity. Indeed, in numerous studies p16 
was scored as positive despite a nuclear and cytoplasmic staining present in $<70 \%$ of malignant cells which probably impacts on the final outcomes. ${ }^{3}$ Admittedly, false-positive cases may be acceptable when it comes to prognostication, but this is unacceptable when the HPV status is used to alter treatment or follow-up. In such a scenario, HPV testing should be accurate and highly specific. It is also striking to note that in some cases p16 is negative despite the presence of E6/E7 mRNA. We observed this situation in two patients (29 and 49). Interestingly, these patients had a smoking history, which is a recognized factor for CDKN2A gene alteration (the gene encoding for p16). Indeed, CNKN2A is one of the most frequently inactivated gene in tobacco induced HNSCC. Studies comparing the genome and epigenome of HPV-driven OPSCC according to their tobacco consumption might provide valuable information.

To improve the accuracy of HPV-driven oropharyngeal squamous cell carcinomas detection, some have advocated detection algorithms that use the strengths of various detection assays used in combination. ${ }^{20,26}$ However, the inclusion of multiple steps to achieve an accurate and reliable HPV status is technically cumbersome, may produce discordant results, and inevitably increases costs and workload. In our study we have also observed that the use of in situ hybridization to detect HPV-DNA in combination with p16, which is one of the two algorithms that are recommended to accurately identify HPV-driven oropharyngeal squamous cell carcinomas, may lead to false negative results. Indeed, we found four cases that were positive for p16 immunohistochemistry and negative for HPVDNA in situ hybridization, suggesting that in these cases p16 overexpression was related to non-viral mechanisms and that these tumors were not HPV-driven. However, HR-HPV mRNAs were found in these four cases attesting that they were HPV-induced. This highlights that the lack of sensitivity of HPV-DNA in situ hybridization is a real problem even when it is used within a diagnostic algorithm.

For these reasons, the RNAscope HPV-test could replace our current tools to precisely identify HPVdriven oropharyngeal squamous cell carcinomas.

Finally, several authors have shown that estimates of disease-specific survival and overall survival by the RNAscope HPV-test were comparable to other assays. ${ }^{13,14,22,23}$

To conclude, the RNAscope HPV-test is a promising assay with four main advantages: (1) it identifies HPV-driven cancers in a single assay as it detects clinically relevant HPV-infections, (2) it permits visualization of viral transcripts in tissues and thus reduce the risk of false positive results, which is a potential risk with contamination of PCR based assays, (3) it is technically feasible for routine testing in clinical laboratories as it uses formalin-fixed paraffin-embedded tissue sections, and (4) its results are easy to interpret.

The RNAscope HPV-test performance against qRT-PCR was very good as demonstrated in three independent studies including a total of 167 OPSCC samples. Explaining the few discrepant cases will be interesting. Before adoption of the RNAscope HPVtest into clinical practice could be formally advocated, this test requires mandatory approval as an in vitro diagnostic device.

\section{Disclosure/conflict of interest}

The RNA in situ hybridization assays (the RNAscope HPV-test) were funded and performed by the coauthors from Advanced Cell Diagnostics (MH and X-JM) who have stocks in this company and may profit by use of this test. All the other tests and the final analyses were performed at the Gustave Roussy Cancer Center. Remaining authors declare no conflict of interest.

\section{References}

1 Marur S, D’Souza G, Westra WH et al. HPV-associated head and neck cancer: a virus-related cancer epidemic. Lancet Oncol 2010;11:781-789.

2 Van Houten VM, Snijders PJ, van den Brekel MW et al. Biological evidence that human papillomaviruses are etiologically involved in a subgroup of head and neck squamous cell carcinomas. Int J Cancer 2001;93: 232-235.

3 Mirghani $\mathrm{H}$, Amen F, Moreau F et al. Human papilloma virus testing in oropharyngeal squamous cell carcinoma: what the clinician should know. Oral Oncol 2014;50:1-9.

4 Snijders PJ, van den Brule AJ, Meijer CJ. The clinical relevance of human papillomavirus testing: relationship between analytical and clinical sensitivity. J Pathol 2003;201:1-6.

5 Gillison ML, Broutian T, Pickard RK et al. Prevalence of oral HPV infection in the United States, 2009-2010. JAMA 2012;307:693-703.

6 Schlecht NF, Brandwein-Gensler M, Nuovo GJ et al. A comparison of clinically utilized human papillomavirus detection methods in head and neck cancer. Mod Pathol 2011;24:1295-1305.

7 Schache AG, Liloglou T, Risk JM et al. Evaluation of human papilloma virus diagnostic testing in oropharyngeal squamous cell carcinoma: sensitivity, specificity, and prognostic discrimination. Clin Cancer Res 2011;17:6262-6271.

8 Weinberger PM, Yu Z, Haffty BG et al. Prognostic significance of p16 protein levels in oropharyngeal squamous cell cancer. Clin Cancer Res 2004;10: 5684-5691.

9 Lewis JS Jr. p16 Immunohistochemistry as a standalone test for risk stratification in oropharyngeal squamous cell carcinoma. Head Neck Pathol 2012;6:75-82.

10 Adelstein DJ, Ridge JA, Gillison ML et al. Head and neck squamous cell cancer and the human papillomavirus: summary of a National Cancer Institute State of 
the Science Meeting, November 9-10, 2008, Washington, D.C. Head Neck 2009;31:1393-1422.

11 Wasylyk B, Abecassis J, Jung AC. Identification of clinically relevant HPV-related HNSCC: in p16 should we trust? Oral Oncol 2013;49:33-37.

12 Rietbergen MM, Snijders PJ, Beekzada D et al. Molecular characterization of p16-immunopositive but HPV DNA-negative oropharyngeal carcinomas. Int J Cancer 2014;134:2366-2372.

13 Schache AG, Liloglou T, Risk JM et al. Validation of a novel diagnostic standard in HPV-positive oropharyngeal squamous cell carcinoma. Br J Cancer 2013;108: 1332-1339.

14 Bishop JA, Ma XJ, Wang $\mathrm{H}$ et al. Detection of transcriptionally active high-risk HPV in patients with head and neck squamous cell carcinoma as visualized by a novel E6/E7 mRNA in situ hybridization method. Am J Surg Pathol 2012;36:1874-1882.

15 Livak KJ, Schmittgen TD. Analysis of relative gene expression data using real-time quantitative PCR and the 2(-Delta Delta C(T)) Method. Methods 2001;25: 402-408.

16 Chaturvedi AK, Engels EA, Pfeiffer RM et al. Human papillomavirus and rising oropharyngeal cancer incidence in the United States. J Clin Oncol 2011;29: 4294-4301.

17 Garnaes E, Kiss K, Andersen L et al. A high and increasing HPV prevalence in tonsillar cancers in Eastern Denmark, 2000-2010: The largest registrybased study to date. Int J Cancer 2015;136:2196-2203.

18 Ang KK, Harris J, Wheeler R et al. Human papillomavirus and survival of patients with oropharyngeal cancer. N Engl J Med 2010;363:24-35.

19 Fakhry C, Westra WH, Li S. Improved survival of patients with human papillomavirus-positive head and neck squamous cell carcinoma in a prospective clinical trial. J Natl Cancer Inst 2008;100:261-269.

20 Smeets SJ, Hesselink AT, Speel EJ et al. A novel algorithm for reliable detection of human papillomavirus in paraffin embedded head and neck cancer specimen. Int J Cancer 2007;121:2465-2472.

21 Gao G, Chernock RD, Gay HA et al. A novel RT-PCR method for quantification of human papillomavirus transcripts in archived tissues and its application in oropharyngeal cancer prognosis. Int J Cancer 2013;132: 882-890.

22 Ukpo OC, Flanagan JJ, Ma XJ et al. High- risk human papillomavirus E6/E7 mRNA detection by a novel in situ hybridization assay strongly correlates with p16 expression and patient outcomes in oropharyngeal squamous cell carcinoma. Am J Surg Pathol 2011;35: 1343-1350.

23 Lewis JS Jr, Ukpo OC, Ma XJ et al. Transcriptionallyactive high-risk human papillomavirus is rare in oral cavity and laryngeal/hypopharyngeal squamous cell carcinomas-a tissue microarray study utilizing E6/E7 mRNA in situ hybridization. Histopathology 2012;60: 982-991.

24 Wang F, Flanagan J, Su N et al. RNAscope: a novel in situ RNA analysis platform for formalin-fixed, paraffin-embedded tissues. J Mol Diagn 2012;14: 22-29.

25 Lingen MW, Xiao W, Schmitt A et al. Low etiologic fraction for high-risk human papillomavirus in oral cavity squamous cell carcinomas. Oral Oncol 2013;49:1-8.

26 Singhi AD, Westra WH. Comparison of human papillomavirus in situ hybridization and p16 immunohistochemistry in the detection of human papillomavirus-associated head and neck cancer based on a prospective clinical experience. Cancer 2010;116:2166-2173. 